Marc Nicolas Sommer

\title{
Der ästhetische Gemeinsinn als ursprüngliche Vermittlung von Natur und Freiheit
}

1

Kants kritisches Projekt lässt sich in seiner systematischen Fluchtlinie als groß angelegte Bewältigung des Antagonismus von Natur und Freiheit verstehen. In einer Kritik der Vermögen des menschlichen Geistes sollen Grenzen und Möglichkeiten der Naturerkenntnis im Rahmen strenger Wissenschaftlichkeit bestimmt und zugleich Möglichkeit und Wirklichkeit der menschlichen Freiheit erwiesen werden. Zwar ist das Sachinteresse nur in der Kritik der praktischen Vernunft von unmittelbar moralphilosophischem Gewicht, aber auch das jeweilige Sachinteresse der ersten und der dritten Kritik lässt sich vom letztlich moralphilosophischen Problem des Verhältnisses von Freiheit und Notwendigkeit verstehen. Wenn Otfried Höffe in Bezug auf die Kritik der reinen Vernunft der von einem eher erkenntnistheoretischen Leitinteresse ausgehenden „orthodoxen“ Lesart eine „heterodoxe“, gar „häretische Lektüre“ gegenüberstellt, die das Leitinteresse als moralisch-praktisches bestimmt, so kann er sich dabei auf Geist und Buchstaben der kantischen Philosophie stützen. ${ }^{1}$ Besonders der „Kanon der reinen Vernunft" in der Methodenlehre der Kritik der reinen Vernunft benennt das moralisch-praktische Interesse in dieser Phase. ${ }^{2}$ Auch in der dritten Kritik lässt sich ein moralisch-praktisches Leitinteresse ausmachen, das sich wiederum in der Methodenlehre, diesmal zur teleologischen Urteilskraft, am deutlichsten herauskristallisiert. $^{3}$

Unter dem Leitinteresse am Verhältnis von Natur und Freiheit hat jede Kritik eine spezifische Funktion: Während die erste Kritik die Möglichkeit der Freiheit inmitten einer determinierten Natur erweist, nimmt die zweite Kritik die Bestimmung des Willens aus reiner praktischer Vernunft vor; die dritte Kritik schließlich

1 Höffe, Otfried: Urteilskraft und Sittlichkeit. Ein moralischer Rückblick auf die dritte Kritik. In: Immanuel Kant. Kritik der Urteilskraft. Hg. v. Otfried Höffe. Berlin 2008, 351-366, 351.

2 Vgl. Kant: KrV, A 801/B 829.

3 Vgl. Kant: KU, AA 05: 447.14-462.10.

Marc Nicolas Sommer, Universität Basel, marc.sommer@unibas.ch

https://doi.org/10.1515/9783110467888-313 
soll zeigen, dass sich die vom freien Willen gesetzten Zwecke auch in der Natur verwirklichen lassen. Spekulatives Interesse und praktisches Interesse der Vernunft stehen nicht unverbunden nebeneinander; in der Frage nach der Möglichkeit der Verwirklichung der aus Freiheit gesetzten Zwecke in der Natur peilt die Vernunft die systematische Einheit ihrer beiden Interessen an. ${ }^{4}$ Diesem Projekt ist die Kritik der Urteilskraft verpflichtet: Sie erweist die Möglichkeit eines Übergangs von der Freiheit zur Natur, indem sie Verhältnisse zur Natur untersucht, in denen die Natur als uns entgegenkommend und als empfänglich für unser Wirken erscheint. In dieser Fluchtlinie behandelt das Werk die ästhetische Beziehung zur Natur sowie die wissenschaftliche Untersuchung der organischen Natur - Beziehungen, in denen die Natur in jeweils spezifischer Form als zweckgerichtet und damit als empfänglich für Zwecke gedacht wird. Die Schönheit als Symbol der Sittlichkeit und die teleologische Betrachtung der organischen Natur sind dabei nur die markantesten Nachweise einer Empfänglichkeit der Natur für die Realisierung sittlicher Zwecke. Die Vermittlung von Natur und Freiheit geschieht in der Kritik der Urteilskraft jedoch schon auf einer tieferen, einerseits basaleren, andererseits aber grundlegenden Ebene: Bereits im Geschmacksurteil finden wir eine Vermittlung von Natur und Freiheit, die uns auf eine Übereinstimmung unseres Erkenntnisvermögens überhaupt mit der Welt verweist. Grundlegend ist die Vermittlung, weil erst durch sie erwiesen wird, dass unser Erkenntnisvermögen der Natur überhaupt angemessen ist. Den Nachweis dieser Angemessenheit findet Kant in der Untersuchung des Schönen. Schon um 1771 schreibt er in einer Notiz: „Die Schöne Dinge zeigen an, daß der Mensch in die Welt passe und selbst seine Anschauung der Dinge mit den Gesetzen seiner Anschauung stimme. " ${ }^{\text {In }}$ In der Kritik der Urteilskraft, so die These, die ich im Folgenden erläutern möchte, begründet Kant diese Behauptung in einer transzendentallogischen Untersuchung des Geschmacksurteils. Dieses verweist auf eine unwillkürliche Verbundenheit unseres Erkenntnisvermögens mit der Natur, die Kant als ästhetischen Gemeinsinn versteht. Um diese These zu entwickeln, muss zweierlei erwiesen werden: Erstens, dass der im Rahmen des Geschmacksurteils angesprochene Gemeinsinn sich auch als Sinn für die Gemeinschaft mit der Natur verstehen lässt; und zweitens, dass die das Geschmacksurteil bestimmende Lust eine Lust an der Affinität unseres Erkenntnisvermögens zur Natur ist.

4 Vgl. Hutter, Axel: Das Interesse der Vernunft. Kants ursprüngliche Einsicht und ihre Entfaltung in den transzendentalphilosophischen Hauptwerken. Hamburg 2003, 90.

5 Kant: HN, AA 16: 127.13-15. 
Die Urteilskraft ist unter all den von Kant untersuchten Vermögen das rätselhafteste; zwar gehört sie neben Verstand und Vernunft zu den oberen Erkenntnisvermögen, verfügt also über Prinzipien a priori; dennoch hat sie kein eigentümliches Gebiet. Ein Gebiet (ditio) ist die kleinste territoriale Einheit der Begriffe, nämlich die Einheit, innerhalb derer Begriffe gesetzgebend sind. Gebiete gibt es nur zwei: das, in dem die Naturbegriffe und damit der Verstand gesetzgebend sind; und das, in dem der Freiheitsbegriff und somit die Vernunft gesetzgebend ist. Angesichts dieser Einteilung besteht die Funktion der Urteilskraft in der Vermittlung zwischen den beiden Gebieten unseres Erkenntnisvermögens. ${ }^{6}$ In dieser Vermittlungsleistung erschöpft sich ihre Kompetenz keineswegs; so hat sie nicht bloß auf makrologischer Ebene die beiden Systemhälften in einen Zusammenhang zu bringen, sondern vermittelt bereits in kleinerem Maßstab zwischen zwei heterogenen Vermögen, die gerade in ihrer jeweiligen Eigentümlichkeit in eine Übereinstimmung gebracht werden müssen. Prominent ist der Fall der Urteilskraft, die in der Kritik der reinen Vernunft im Schematismus der reinen Verstandesbegriffe am Werk ist. Die Urteilskraft soll hier mittels der transzendentalen Schemata zwischen den intellektuellen Verstandesbegriffen und den sinnlichen Anschauungen vermitteln. Sie sorgt dafür, dass in der Anwendung der Begriffe auf Anschauung nicht Willkür herrscht, sondern Gesetzmäßigkeit, nämlich die des Verstandes.

In der Kritik der Urteilskraft untersucht Kant nun einen Fall, in dem die Urteilskraft eine ähnliche Vermittlung zwischen zwei Vermögen vollbringt, allerdings ohne selbst unter der Leitung eines bestimmten Vermögens zu stehen. Im Geschmacksurteil über das Schöne agieren die in Frage stehenden Vermögen der Verstand und die Einbildungskraft - in freiem Zusammenspiel und kommen in eine harmonische Übereinstimmung, die als Gefühl der Lust empfunden wird. Diese Lust muten wir jedermann zu, wenn wir ein Geschmacksurteil fällen. Die Allgemeingültigkeit des Geschmacksurteils hat nach Kant gerade darin ihre Eigentümlichkeit, dass hier nicht ein empirischer Begriff, sondern ein Gefühl der Lust jedermann zugemutet werden soll. ${ }^{7}$ Diese Zumutung beruht auf dem, was Kant einen Gemeinsinn nennt.

Was der Begriff ,Gemeinsinn“ alles umfasst, ist in der Kritik der Urteilskraft nicht immer eindeutig, und Kants Bezugnahme auf den gesunden Menschenverstand, der ebenfalls Gemeinsinn, oder sensus communis genannt wird, ist dem

6 Kant: KU, AA 05: 174.01-179.15.

7 Kant: KU, AA 05: 191.06-11. 
Verständnis des im Geschmacksurteil in Frage stehenden Gemeinsinns wenig zuträglich. Begriffsgeschichtlich lässt sich der sensus communis auf zwei Quellen zurückführen: Anschließend an Cicero kann der Gemeinsinn als ein von einer Gruppe von Menschen geteilter, mithin intersubjektiver Sinn verstanden werden; oder er kann, anschließend an Aristoteles, als Zusammenfassung der einzelnen Sinne im Subjekt, mithin als intrasubjektiver Sinn verstanden werden. In Kants Analyse des Geschmacksurteils spielen beide Erbschaften eine Rolle. ${ }^{8}$ Daneben gibt es bei Kant jedoch noch eine dritte Bedeutungsschicht im Gemeinsinn, in der er als Sinn für die Gemeinschaft mit der Natur verstanden werden kann. Um diese dritte Schicht freizulegen, sind die erste und die zweite Bedeutungsschicht des ästhetischen Gemeinsinns in ihrem Verhältnis zu rekonstruieren.

\section{3}

Kant führt den Begriff des Gemeinsinns im § 20 der Kritik der Urteilskraft ein; der Titel dieses Paragraphen bestimmt den Gemeinsinn als „Die Bedingung der Möglichkeit, die ein Geschmacksurtheil vorgibt". ${ }^{9}$ Mithin ist der Gemeinsinn im Bereich des Transzendentalen zu verorten. Wie an anderen Stellen seines Werks geht Kant von einem Faktum aus und sucht die transzendentalen Bedingungen, die dieses Faktum möglich machen. So sagt er im § 22: „Diese unbestimmte Norm eines Gemeinsinns wird von uns wirklich vorausgesetzt; das beweist unsere Anmaßung, Geschmacksurtheile zu fällen. " ${ }^{10}$ Die transzendentale Stoßrichtung der Frage nach einem Gemeinsinn verbietet es, diesen anthropologisch zu verstehen; wenn auch der von Kant eine „Episode“ genannte Exkurs über die Maximen des gesunden Menschenverstandes eine solche Lektüre nahelegen mag, zumal dieselben Maximen auch in der Anthropologie in pragmatischer Hinsicht behandelt werden, ${ }^{11}$ so wird in den übrigen Teilen des $\S 40$ deutlich, dass die Episode nur illustrativen Zwecken dient. Bereits der Titel „Vom Geschmacke als einer Art von sensus communis“ legt nahe, die Gegenüberstellung von Geschmack als sensus communis aestheticus und dem gemeinen Menschenverstand als sensus communis

8 Vgl. Wenzel, Christian Helmut: Gemeinsinn und das Schöne als Symbol des Sittlichen. In: Die Vollendung der Transzendentalphilosophie in Kants „Kritik der Urteilskraft“. Hg. v. Reinhard Hiltscher, Stefan Klingner und David Süß. Berlin 2006, 125-139, 130; Kohler, Georg: Gemeinsinn oder: Über das Gute am Schönen. Von der Geschmackslehre zur Teleologie (\$§ 39-42). In: Immanuel Kant. Kritik der Urteilskraft. Hg. v. Otfried Höffe. Berlin 2008, 137-150, 144.

9 Kant: KU, AA 05: 237.32-33.

10 Kant: KU, AA 05: 239.34-35.

11 Vgl. Kant: Anth, AA 07: 200.31-37. 
logicus dahingehend zu verstehen, dass der Geschmack als transzendentallogisch verstandener Gemeinsinn über einen Vergleich mit dem empirischen Gemeinsinn in seiner Eigentümlichkeit dargestellt werden soll. ${ }^{12}$ Beiden gemeinsam ist die Reflexion, die das eigene Urteil auf die möglichen Urteile anderer bezieht; im Falle des ästhetischen Gemeinsinns ist diese Reflexion jedoch nicht empirisch, da sie das Urteil nicht an tatsächlichen oder möglichen Urteilen einer bestimmten Gemeinschaft abgleicht, sondern das Urteil ,gleichsam an die gesamte Menschenvernunft“ hält. ${ }^{13}$ Das Vergleichen der Urteile ist beim ästhetischen Gemeinsinn, wie Lyotard sagt, ,eidetisch'. Es gehe darum, durch ,imaginäre Variationen“ ein reines ästhetisches Urteil zu fällen, es von allem empirischen Gefühl zu reinigen. ${ }^{14}$ Wenn wir also im ästhetischen Gemeinsinn ein auf Ciceros Begriffsgebrauch verweisendes, intersubjektives Moment behaupten, so muss dieses Moment transzendental, in Bezug auf die von allen Menschen geteilte Vernunft, gedacht werden.

Was Kant voraussetzt, ist nach den $\S \S 38$ und 39 zunächst nur dies: dass die Lust, die Bestimmungsgrund des ästhetischen Urteils ist, bei allen Menschen auf denselben Bedingungen beruht, weil diese Bedingungen

[...] subjective Bedingungen der Möglichkeit einer Erkenntniß überhaupt sind, und die Proportion dieser Erkenntnisvermögen, welche zum Geschmack erfordert wird, auch zum gemeinen und gesunden Verstande erforderlich ist, den man bei jedermann voraussetzen darf. $^{15}$

Kant setzt also nicht bloß voraus, dass die Erkenntnisvermögen in jedem Menschen dieselben sind, sondern auch dass die im Geschmacksurteil erreichte Proportion der Vermögen bei allen Menschen anzutreffen ist. Kant muss diese Voraussetzung machen, da die Proportion der Vermögen im Geschmacksurteil nicht begrifflich mitteilbar ist, wie im Fall der Erkenntnis nach Begriffen; die Proportion ist nur als Gefühl der Lust bestimmbar und dieses Gefühl der Lust ist das, was für die Kommunizierbarkeit des Geschmacksurteils in allen Menschen vorausgesetzt wird.

12 ,Art` ist hier also, wie Jens Kulenkampff sagt, im Sinne von ,Spezies‘ zu verstehen. Kulenkampff, Jens: „Vom Geschmacke als einer Art von sensus communis“ - Versuch einer Neubestimmung des Geschmacksurteils. In: Autonomie der Kunst? Zur Aktualität von Kants Ästhetik. Hg. v. Andrea Esser. Berlin 1995, 25-48, 26.

13 Kant: KU, AA 05: 293.33-34.

14 Lyotard, Jean-François: Sensus Communis. In: Le Cahier (Collège international de philosophie) 3 (1987), 67-87, 86.

15 Kant: KU, AA 05: 292.36-293.03. 
Das Gefühl der Lust ist aber in bestimmter Hinsicht bereits selbst ein Gemeinsinn; denn Kant versteht unter dem Gemeinsinn, wie er in § 20 sagt, „keinen äußeren Sinn, sondern die Wirkung aus dem freien Spiel unserer Erkenntnißkräfte“. ${ }^{16}$ Die Wirkung aus dem freien Spiel der Erkenntniskräfte ist eine bestimmte, ästhetische Proportion von Verstand und Einbildungskraft, die als Gefühl der Lust wahrgenommen wird. Kant geht dabei von verschiedenen möglichen Proportionen dieser Vermögen aus; das Eigentümliche der Lust erzeugenden, ästhetischen Proportion ist einerseits darin zu suchen, dass sie „die zuträglichste für beide Gemüthskräfte in Absicht auf Erkenntniß [...] überhaupt ist““, ${ }^{17}$ und andererseits der Umstand, dass ihre Harmonie in einem freien Zusammenspiel der Erkenntniskräfte entsteht. ${ }^{18}$ Der Gemeinsinn als Gefühl der Lust, als das die in freiem Spiel entstandene Harmonie der Vermögen sich bemerkbar macht, ist mithin auch ein intrasubjektiver Sinn und verweist damit auf die aristotelische Wurzel des Begriffs: Während aber der Ausdruck „koine aisthesis“ bei Aristoteles als innerer Sinn die Zusammenfassung der Wahrnehmungsinhalte der äußeren Sinne meint, ${ }^{19}$ benutzt Kant den Begriff ,Sinn` hier nur in übertragener Bedeutung; wie er im $\S 40$ ausführt, versteht er unter ,Sinn“ nur eine „Wirkung der bloßen Reflexion auf das Gemüth“. ${ }^{20}$ Der Gemeinsinn ist kein eigentlicher Sinn, sondern nur das sinnliche Analogon der transzendentalen Euphonie der Vermögen. ${ }^{21}$

Damit wird auch deutlich, in welchem Verhältnis die beiden begriffsgeschichtlichen Erbschaften des Gemeinsinnes bei Kant stehen: Wenn wir Geschmacksurteile fällen, setzen wir zunächst einen intersubjektiven Gemeinsinn voraus; wir unterstellen, dass jedermann beim Anblick desselben als schön empfundenen Objekts dasselbe Gefühl der Lust empfindet. Indem wir aber dasselbe Gefühl der Lust bei jedermann voraussetzen, setzten wir zugleich einen intrasubjektiven Gemeinsinn voraus: Denn das Gefühl der Lust ist ja in anderer Hinsicht der Gemeinsinn selbst, als die bloß gefühlte ästhetische Harmonie der Erkenntnisvermögen. Was wir also als intersubjektiven Gemeinsinn dem Geschmacksurteil zugrunde legen, ruht selber auf der Voraussetzung eines intrasubjektiven Gemeinsinnes, nämlich einer aus freiem Spiel resultierenden har-

16 Kant: KU, AA 05: 238.13-14.

17 Kant: KU, AA 05: 238.32-239.02.

18 Kant: KU, AA 05: 217.21-23.

19 Aristoteles: De Anima, Buch III, 425a 27.

20 Kant: KU, AA 05: 295.24-25.

21 Lyotard, Jean-François: Sensus Communis. In: Le Cahier (Collège international de philosophie) 3 (1987), 67-87, 87. 
monischen Proportion der Erkenntniskräfte, die als Gefühl der Lust empfunden wird.

\section{4}

Die dritte Bedeutungsschicht des Gemeinsinns bei Kant erschließt sich uns, wenn wir fragen, warum das harmonische Zusammenspiel der Vermögen als Gefühl der Lust empfunden wird. Etwas wird als schön empfunden, weil es die bei jeder Erkenntnis involvierten Vermögen Verstand und Einbildungskraft in ein freies Zusammenspiel bringt, das in einer harmonischen Proportion mündet. Wir haben diese Proportion bereits anhand zweier Merkmale bestimmt: Sie entsteht erstens nur durch ein freies, das heißt, nicht durch Begriffe geleitetes Zusammenspiel von Verstand und Einbildungskraft; die in diesem Zusammenspiel erreichte harmonische Proportion ist zweitens den beiden ins Spiel getreten Vermögen die $z u$ träglichste.

Was die in Frage stehende ästhetische Proportion der Vermögen von anderen Proportionen unterscheidet, ist einerseits die Freiheit des Zusammenspiels, andererseits der Grad der Zuträglichkeit für die einzelnen Vermögen. Eine Harmonie, im Sinne eines bestimmten Zusammenstimmens der Vermögen, ist nicht ein eigentümliches Merkmal der ästhetischen Proportion, sondern ist „zu einem Erkenntnisse überhaupt erforderlich“. ${ }^{22}$ Eine Harmonie der Vermögen ist Bedingung der Möglichkeit von Erkenntnis überhaupt; würden die Vermögen nicht in geregelten Proportionen zusammenstimmen, so wären die Zusammenhänge zwischen Ideen, Begriffen und Anschauungen willkürlich. Für Gilles Deleuze liegt an dieser Stelle eines der profundesten Probleme der kantischen Philosophie: Wenn die Vermögen ihrer Natur nach verschieden sind, ${ }^{23}$ wie ist es dann zu erklären, dass sie in geregelten Proportionen zusammenstimmen? Es ist, so Deleuze, nicht mit Kants kritischem Programm vereinbar, zwischen den Vermögen eine prästabilierte Harmonie anzunehmen; dieses Programm verlange vielmehr, dass auch diese Harmonie in ihrer Genese nachgezeichnet wird. ${ }^{24}$

Vor diesem Hintergrund lässt sich die ausgezeichnete Stellung der ästhetischen Proportion präzisieren: Sie ist die einzige, in der die Harmonie der Vermögen aus einem freien Zusammenspiel entsteht; so zeigt sie an, dass unsere Vermögen auch ohne Zwang, das heißt, ohne unter der Leitung der Vernunft oder

22 Kant: KU, AA 05: 218.01-03.

23 Kant: KrV, A 271/B 327.

24 Deleuze, Gilles: La philosophie critique de Kant. Paris 2011, $34 \mathrm{f}$. 
des Verstandes zu stehen, zu einer harmonischen Übereinstimmung kommen können. Allerdings ist das freie Spiel der Erkenntniskräfte nicht bloß ein Spiel der Erkenntniskräfte mit sich selbst, sondern es wird in Gang gebracht durch den als schön empfundenen Gegenstand. Obwohl sie an die gegebene Form dieses Gegenstandes gebunden ist, wird die Einbildungskraft dabei nicht in ihrer Freiheit eingeschränkt, da Kant annimmt,

[...] daß der Gegenstand ihr gerade eine solche Form an die Hand geben könne, die eine Zusammensetzung des Mannigfaltigen enthält, wie sie die Einbildungskraft, wenn sie sich selbst frei überlassen wäre, in Einstimmung mit der Verstandesgesetzmäßigkeit überhaupt entwerfen würde. ${ }^{25}$

Das Ausgezeichnete der ästhetischen Stimmung ist also nicht in der freien Harmonie der Vermögen allein zu suchen, sondern im Umstand, dass die freie Harmonie der Vermögen anlässlich von gegebenen Naturgegenständen entsteht. Die durch den konstituierten Gegenstand gegebene Form ist notwendige, aber nicht hinreichende Bedingung einer freien Betätigung der Einbildungskraft, die in einer freien Harmonie zwischen Verstand und Einbildungskraft mündet. Die Harmonie ist deshalb nicht bloß eine intrasubjektive Harmonie der Vermögen, sondern auch eine Harmonie von Einbildungskraft und Natur. Was wir also im Geschmacksurteil als Gefühl der Lust empfinden, ist die gedoppelte Harmonie zwischen Verstand und Einbildungskraft und dieser beiden mit der Natur. ${ }^{26}$ Da der ästhetische Gemeinsinn nichts anderes als dieses Gefühl der Lust ist, können wir die dritte Bedeutungsschicht des Gemeinsinns als freie Übereinstimmung unserer Erkenntnisvermögen mit der Natur bestimmen, mithin den Gemeinsinn auch als Sinn für die Gemeinschaft mit der Natur verstehen. Dieser Gemeinsinn ist das Gefühl einer unwillkürlichen, gleichsam unerzwungenen Affinität unserer Vermögen zur Natur: Deshalb zeigen die schönen Dinge an, dass wir in die Welt passen.

25 Kant: KU, AA 05: 240.28-241.03. Vgl. zum Verhältnis von gegebener Form und ästhetischer Reflexion: Esser, Andrea Marlen: Kunst als Symbol. Die Struktur ästhetischer Reflexion in Kants Theorie des Schönen. München 1997, 159-163. Und zur Freiheit des freien Spiels der Erkenntniskräfte: Stolzenberg, Jürgen: Das freie Spiel der Erkenntniskräfte. Zu Kants Theorie des Geschmacksurteils. In: Kants Schlüssel zur Kritik des Geschmacks. Ästhetische Erfahrung heute Studien zur Aktualität von Kants „Kritik der Urteilskraft“. Hg. v. Ursula Franke. Hamburg 2000, $1-28,8-12$.

26 Vgl. Kant: KU, AA 05: 189.36-190.02. 